ARTICLE

\title{
Long-term respiratory and developmental outcomes in children with bronchopulmonary dysplasia and history of tracheostomy
}

\author{
Chandler A. Annesi $\mathbb{D}^{1}$, Jonathan C. Levin $\mathbb{D}^{2,3,4}$, Jonathan S. Litt ${ }^{3,4}$, Catherine A. Sheils ${ }^{2,4}$ and Lystra P. Hayden $\mathbb{D}^{2,4,5}{ }^{\circ}$
}

(c) The Author(s), under exclusive licence to Springer Nature America, Inc. 2021

\begin{abstract}
OBJECTIVE: The long-term morbidity among children with severe bronchopulmonary dysplasia who require tracheostomy (tBPD) relative to those without tracheostomy (SBPD) is not well characterized. We compared childhood lung function and neurodevelopmental outcomes in tBPD and SBPD.

STUDY DESIGN: Retrospective case-control study of $N=49$ tBPD and $N=280$ sBPD subjects in Boston Children's Hospital Preterm Lung Patient Registry and medical record. We compared NICU course, childhood spirometry, and neurodevelopmental testing. RESULT: tBPD subjects were more likely than sBPD to be Black, have pulmonary hypertension, and have subglottic stenosis. tBPD subjects had lower maximal childhood $\mathrm{FEV}_{1} \%$ predicted $(\beta=-0.14)$ and $\mathrm{FEV}_{1} / \mathrm{FVC}(\beta=-0.08)$; spirometry curves were more likely to suggest fixed extrathoracic obstruction. tBPD subjects had greater cognitive and motor delays $<24$ months, and greater cognitive delays $>24$ months.

CONCLUSION: Compared to subjects with sBPD who did not require tracheostomy, tBPD subjects suffer from increased long-term impairment in respiratory function and neurodevelopment.
\end{abstract}

Journal of Perinatology (2021) 41:2645-2650; https://doi.org/10.1038/s41372-021-01144-0

\section{INTRODUCTION}

In severe cases of bronchopulmonary dysplasia (BPD), patients may be candidates for tracheostomy. It is estimated that 13,000 patients develop severe BPD annually, with 5\% requiring tracheostomy [1, 2]. Tracheostomy is considered in BPD infants when it is anticipated that high-level respiratory support will be needed for a prolonged period of time due to respiratory insufficiency, upper or lower airway abnormalities, or a combination of these [3-6]. The long-term impact of tracheostomy placement on respiratory and neurodevelopmental outcomes is not well established.

All preterm infants are at risk for childhood respiratory morbidity and neurodevelopmental impairment (NDI) with increased likelihood in patients with severe BPD [7]. It is recommended that tracheostomy be placed by term to limit the physical risks associated with repeated intubations as well as decrease the risk of NDI [3]. Although a number studies have examined childhood pulmonary function [8-11] and neurodevelopmental $[12,13]$ outcomes in BPD, few have directly compared BPD patients with history of tracheostomy (tBPD) to severe BPD patients who do not receive tracheostomy (sBPD) [3, 14].

This study examines the childhood respiratory and neurodevelopmental outcomes of severe BPD patients with and without tracheostomy in a large, contemporary US cohort from a single tertiary/quaternary care center. We hypothesize that tBPD will have decreased childhood lung function and worse NDI compared to SBPD patients without tracheostomy.

\section{MATERIALS/SUBJECTS AND METHODS Subject identification}

Subjects were identified using the Boston Children's Hospital $(\mathrm{BCH})$ Preterm Lung Patient Registry [15], active since 2008, with additional subjects added to the tracheostomy group by medical record review. The patient registry included 1068 former preterm infants with gestational age $<37$ weeks who were seen in the pulmonary clinic (August 26, 2020 download). Prospectively enrolled Patient Registry subjects completed informed consent, with a small subset of subjects enrolled under a waiver of consent for retrospective review. Subjects were excluded if they had known genetic or congenital causes of their respiratory disease, including congenital cyanotic heart disease. To expand the population of subjects with BPD and history of tracheostomy, an additional search was performed using i2b2 [16] to identify subjects based on the International Classification of Diseases diagnosis codes in the $\mathrm{BCH}$ electronic medical record.

Severe BPD was defined as having used $\geq 28$ days of respiratory support in the neonatal intensive care unit (NICU) and using ventilation, continuous positive airway pressure, or high flow nasal cannula at 36 weeks corrected gestational age (CGA) [17]. History of tracheostomy placement was identified in the Patient Registry or i 2 b2 search and confirmed by medical record review. Medical record data for subjects' initial NICU admissions were collected including birth characteristics, NICU treatments, and information about NICU discharge. Chart review and BCH HoundDog [18], a natural language processing search tool, was used to identify each subject's relevant comorbidities.

\footnotetext{
${ }^{1}$ Boston University School of Medicine, Boston, MA, USA. ${ }^{2}$ Division of Pulmonary Medicine, Boston Children's Hospital, Boston, MA, USA. ${ }^{3}$ Division of Newborn Medicine, Boston Children's Hospital, Boston, MA, USA. ${ }^{4}$ Department of Pediatrics, Harvard Medical School, Boston, MA, USA. ${ }^{5}$ Channing Division of Network Medicine, Brigham and Women's Hospital, Boston, MA, USA. ${ }^{\bowtie}$ email: Lystra.Hayden@childrens.harvard.edu
}

Received: 20 January 2021 Revised: 20 June 2021 Accepted: 30 June 2021

Published online: 21 July 2021 
Table 1. Demographic and NICU course data in BPD subjects with and without history of tracheostomy.

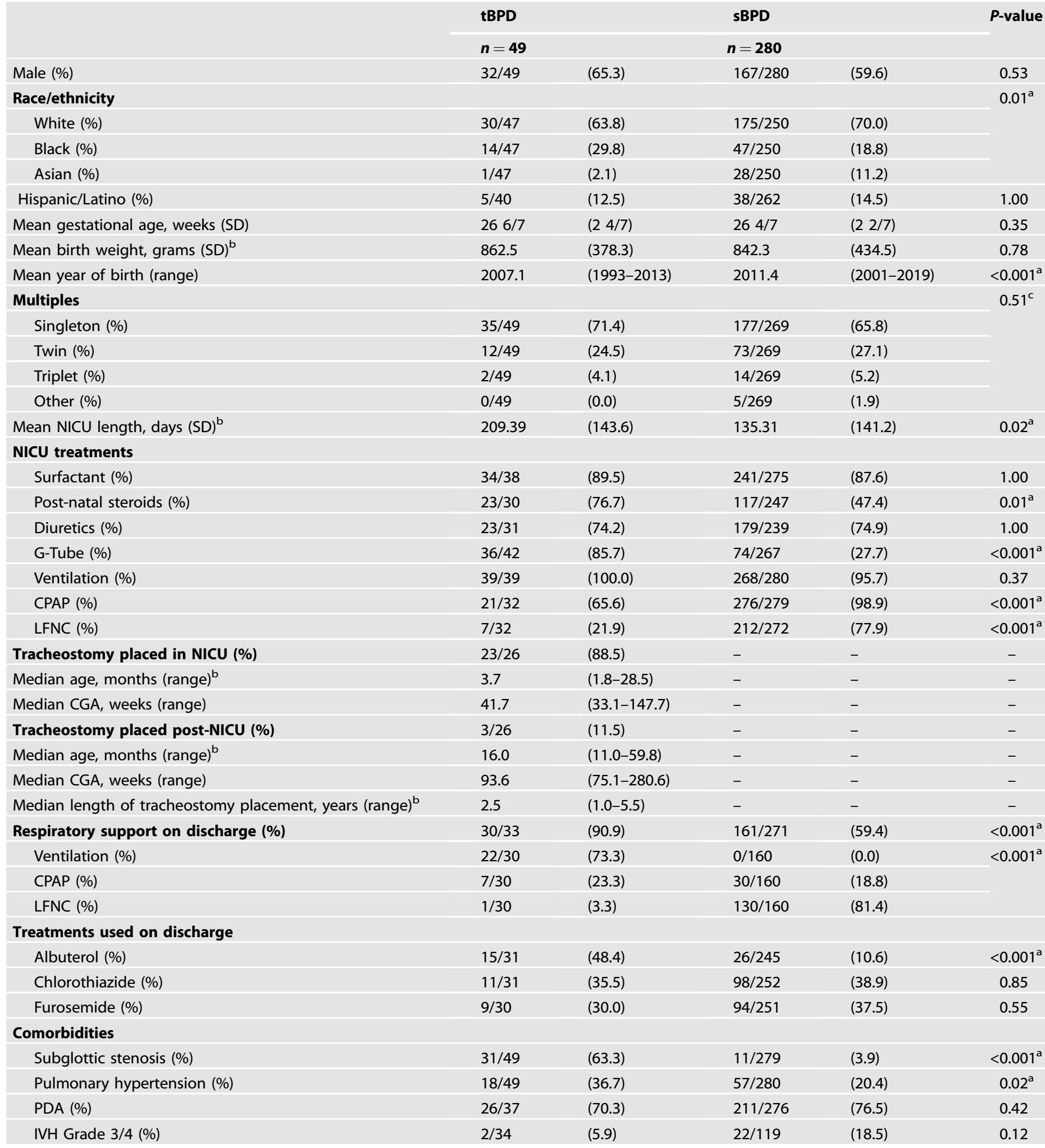

Positive respondents/total respondents.

$B P D$ bronchopulmonary dysplasia, $t B P D$ BPD with history of tracheostomy, $S B P D$ severe BPD without history of tracheostomy, NICU neonatal intensive care unit, G-Tube gastronomy tube, CPAP continuous positive airway pressure, LFNC low flow nasal cannula, PDA patent ductus arteriosus, IVH intraventricular. hemorrhage

a $P<0.05$.

${ }^{\mathrm{b}}$ Response missing for some subjects.

${ }^{\mathrm{c}}$ Analysis comparing singleton birth to non-singleton birth. 
Table 2. Childhood best spirometry results in BPD subjects with and without history of tracheostomy.

\begin{tabular}{|c|c|c|c|c|c|c|c|}
\hline & \multirow{2}{*}{$\begin{array}{l}\text { tBPD } \\
n=23\end{array}$} & \multicolumn{2}{|r|}{ SBPD } & & \multirow[t]{2}{*}{$\beta$} & \multirow[t]{2}{*}{$95 \% \mathrm{Cl}$} & \multirow[t]{2}{*}{$P$-value } \\
\hline & & & $\boldsymbol{n}=\mathbf{5 0}$ & & & & \\
\hline Mean FEV pp $_{1}(S D)^{a}$ & 70.7 & (23.6) & 85.3 & $(14.7)$ & -14.16 & $(-23.64,-4.68)$ & $0.01^{\mathrm{b}}$ \\
\hline Mean FEV $1 /$ FVC (SD) ${ }^{a, c}$ & 72.2 & $(14.1)$ & 81.9 & (8.9) & -8.23 & $(-15.72,-0.74)$ & $0.03^{b}$ \\
\hline
\end{tabular}

$B P D$ bronchopulmonary dysplasia, $t B P D B P D$ with history of tracheostomy, $S B P D$ severe BPD without history of tracheostomy, $F E V_{1}$ forced expiratory volume in one second, FVC forced vital capacity, $p p$ percent predicted.

${ }^{a}$ Controlled for gestational age and multiple gestations.

b $P<0.05$.

b Only includes subjects with acceptable FVC.

\section{Pulmonary function}

Pre-bronchodilator spirometry was performed on a Medisoft SpiroAir machine (Sorinnes, Belgium). Data was recorded in and extracted from Morgan Scientific ComPAS software (Haverhill, MA, USA) [19] and normalized to percent predicted $(\mathrm{pp})$ values using Global Lung Function Initiative equations [20]. Data were assessed for acceptable technique based on modified American Thoracic Society Guidelines as previously described [15]. Spirometry sessions were excluded if they did not meet the criteria for forced expiratory volume in $1 \mathrm{~s}$ $\left(\mathrm{FEV}_{1}\right)$ on at least one effort. For tBPD subjects, only postdecannulation spirometry sessions were included. Childhood best $\mathrm{FEV}_{1} \mathrm{pp}$, forced vital capacity (FVC) pp, and $\mathrm{FEV}_{1} / \mathrm{FVC}$ were analyzed among all spirometry sessions with acceptable technique. Flow volume loops among all subjects were visually assessed and in tBPD subjects were scored in blinded review as presence or absence of flattened expiratory loops, defined as lacking a true peak and with a flattened slope (Supplemental Fig. 1).

\section{Neurodevelopmental assessment}

Medical record search using BCH Clinical Research Informatics Team bioinformatics search tool identified subjects who had visited BCH NICU Growth and Developmental Support Programs (NICU GraDS) for neurodevelopmental follow-up testing using the Bayley Scales of Infant Development-III (Bayley) [21]. The BCH HoundDog [18] natural language processing search tool was used to identify subjects who had completed Bayley testing and medical record review was used to collect individual scores. The subject's CGA at the time of testing was determined. Their composite scores for cognitive abilities and scaled scores for gross motor and fine motor were collected.

\section{Statistical analyses}

Subjects with tBPD were compared to SBPD based on birth characteristics, NICU treatments, NICU comorbidities, NICU discharge information, tracheostomy placement, and decannulation using univariate analysis with chi-square or independent sample ttests. We compared mean Bayley scores for testing done at $<12$ months CGA, 12-23.99 months CGA, and $\geq 24$ months CGA with independent sample t-tests. Bin sizes for different age and domain stratifications were limited and there was insufficient power to perform a regression analysis. Analyses were performed on IBM SPSS Statistics for Macintosh Version 26.0 [22].

Childhood best spirometry was compared using multivariable linear regression adjusted for gestational age and multiple gestations run as singleton vs. non-singleton births. Analysis was performed using R v3.5.2 (2018-12-20) [15].

\section{RESULTS}

Subject demographics and NICU course

A total of 329 subjects were identified for inclusion, 49 tBPD and 280 sBPD (Table 1). Among tBPD subjects, 39 were from the
Patient Registry and 10 were identified by medical record review. All sBPD subjects were from the Patient Registry. Compared to those with sBPD, tBPD subjects were more likely to be of Black race and, on average, were born at an earlier birth year (consistent with the use of medical review to include additional subjects, including some born before the Patient Registry was started). There were no differences in gender, Hispanic/Latino ethnicity, gestational age, birth weight, or multiple gestation status between the two groups. Analysis of NICU treatments showed significantly increased rates of post-natal steroid use, gastrostomy tube placement, respiratory support in the NICU and on discharge, and length of NICU course in tBPD subjects. On discharge, there was an increased prescription rate of albuterol of tBPD subjects compared to SBPD subjects and no differences in the prescribing rates of chlorothiazide and furosemide. There were increased comorbidities of subglottic stenosis and pulmonary hypertension among tBPD subjects compared to those with SBPD, while no significant differences were found in the percent of subjects diagnosed with Grade 3 or 4 intraventricular hemorrhage or patent ductus arteriosus. Of note, there was no difference in any comorbidity diagnoses between tBPD patients found through the Patient Registry compared to those in the medical record (Supplemental Table 1). Significantly more tBPD subjects who were diagnosed with cerebral palsy compared to SBPD subjects, though the analysis had notable limitations as described in the Supplementary Information, pg 1.

Among tBPD subjects, $88.5 \%$ had tracheostomies placed in the NICU at a median age of 3.7 months and median CGA of 41.7 weeks (Table 1). There were three tBPD subjects (11.5\%) who had tracheostomies placed after NICU discharge, at a median age of 16.0 months and median CGA of 93.6 weeks, in the setting of upper airway abnormalities and respiratory infections as described in the Supplemental Material. Decannulation occurred after a median length of 2.5 years. Age at tracheostomy placement was not available for 23 of the 49 tBPD subjects and duration of tracheostomy placement was not available for 10 of the 49 tBPD subjects due to patients moving their medical home and incomplete medical record information.

\section{Pulmonary function}

Among the subset of subjects with acceptable $\mathrm{FEV}_{1}$ data available, 23 with $\mathrm{ABPD}$ and 50 with SBPD, demographics and comorbidities were compared (Supplemental Table 2). Compared to the overall population, this subset demonstrated a significant difference in the proportion of multiple gestations with tBPD subjects more likely to be a singleton birth $(87.0 \%)$ than sBPD subjects $(58.0 \%$, $P=0.02$ ). There were no significant differences between tBPD and sBPD subjects in race or rates of pulmonary hypertension. Notably, tBPD subjects were significantly older at the time of their childhood best spirometry, likely related to their earlier birth year and longer longitudinal follow-up in pulmonary clinic relative to sBPD subjects. 
(a)

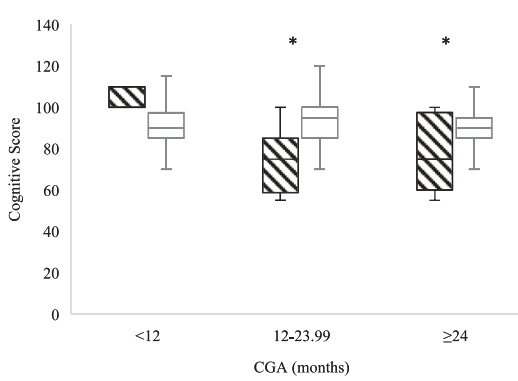

(b)

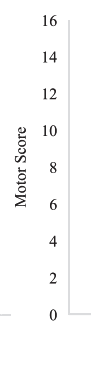

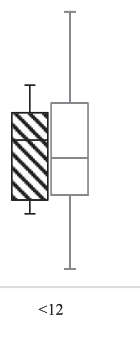

(c)

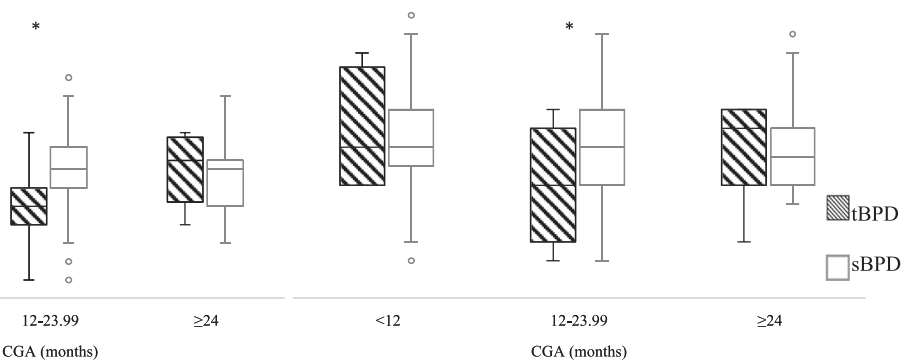

Fig. 1 Distribution of neurodevelopmental testing scores in BPD subjects with and without history of tracheostomy based on corrected gestational age. Bayley scores presented for (a) cognitive, (b) gross motor, and (c) fine motor. ${ }^{*} P<0.05$. CGA corrected gestational age, BPD bronchopulmonary dysplasia, tBPD BPD with history of tracheostomy, sBPD severe BPD without history of tracheostomy.

History of tracheostomy was associated with a significantly lower mean childhood best $\mathrm{FEV}_{1} \mathrm{pp}$, when controlling for gestational age and multiple gestations (tBPD 70.7\%, sBPD $85.3 \% ; \beta=-14.16, P=0.01$ ) (Table 2). Among $17 \mathrm{tBPD}$ and 30 sBPD subjects with acceptable FVC pp data available, mean $\mathrm{FEV}_{1} / \mathrm{FVC}$ was significantly lower among tBPD subjects (tBPD 72.2, sBPD 81.9; $\beta=-8.23, P=0.03$ ), while FVC pp alone approached but did not reach significance for difference between the two groups (tBPD 94.5\%, sBPD 107.0\%; $\beta=-13.58, P=0.06$ ).

There were no sBPD subjects in the spirometry subset with subglottic stenosis; 19 of the 23 tBPD subjects carried this diagnosis (tBPD 82.6\%, sBPD 0.0\%, $P<0.001$ ) (Supplemental Table 2). Among tBPD subjects, flow-volume loops assessed in blinded review identified four subjects with flattening all of whom were diagnosed with subglottic stenosis (21.1\%) (Supplemental Fig. 1).

\section{Neurodevelopment assessment}

A total of 125 subjects, 16 tBPD and 109 sBPD, completed neurodevelopmental Bayley testing (Supplemental Table 3). Compared to the overall cohort, there was no longer any difference in the proportion of patients of Hispanic/Latino ethnicity or the rate of pulmonary hypertension between the two groups.

Comparison of composite Bayley scores in subjects up to three years of age across three neurodevelopmental domains was completed among $11 \mathrm{tBPD}$ and 64 sBPD subjects with cognitive testing, 12 tBPD and 103 sBPD subjects with gross motor testing, and 12 tBPD and 100 sBPD subjects with fine motor testing (Supplemental Table 4, Fig. 1). Test scores for each subject by CGA at the time of the test were reported, and subjects with data from more than one testing session can appear in multiple bins. In univariate analysis cognitive, fine motor, and gross motor domain scores were significantly lower in tBPD subjects at 12-23.99 months CGA, and cognitive scores were significantly lower at $\geq 24$ months CGA.

\section{DISCUSSION}

To the best of our knowledge, this is the largest study to assess long-term respiratory and neurodevelopmental outcomes in tBPD subjects compared to SBPD subjects without tracheostomy, including 49 tBPD and 280 sBPD subjects. Compared to sBPD infants, those with tBPD were more likely to of Black race, have longer more complicated NICU courses, and have increased comorbidities including pulmonary hypertension and subglottic stenosis. In examining long-term outcomes, tBPD subjects had decreased childhood pulmonary function, and a subset of subjects with subglottic stenosis demonstrated fixed airway obstruction suggesting an extrathoracic cause contributing to decreased $\mathrm{FEV}_{1}$. In neurodevelopmental testing up to three years of age, tBPD subjects had lower scores across cognitive, gross motor, and fine motor domains. This study demonstrates the long-term increase in respiratory and neurodevelopmental comorbidity in tBPD patients compared to those with SBPD who do not require tracheostomy, which likely reflects the degree of overall disease severity in this population rather than the direct impact of tracheostomy placement itself.

Patients with tBPD represent an extreme of severe respiratory morbidity, and their long-term outcomes are an important area of research [14, 23-25]. Similar to what is seen in this cohort, previous studies have demonstrated that tBPD patients have increased gastronomy tube placement [14] and post-natal steroids administration [23] compared to sBPD patients, and are more likely to be diagnosed with comorbidities including pulmonary hypertension [24, 25]. Earlier studies have looked at outcomes in tBPD patients, but only neurodevelopmental outcomes were directly compared with a similar SBPD group [3, 14]. Comparing long-term outcomes in severe BPD patients who are a part of these two groups can help providers and families understand the course of tBPD patients.

In this study of severe BPD subjects $15 \%$ received tracheostomy. Previous studies have reported that tracheostomy is placed in 10$50 \%$ of all BPD patients, with wide variability based on institutional practices $[2,4,26-29]$. The most common reason for tracheostomy placement in premature infants is prolonged ventilator dependence followed by large airway disease including subglottic stenosis and tracheobronchomalacia [4, 23, 24]. We found that the indication for tracheostomy was complicated by prolonged ventilator dependence and the possible presence of large airway disease, making a single indication difficult to delineate. Tracheostomies in our cohort were placed at an average age of 3.7 months in the $88.5 \%$ of subjects who had them placed before NICU discharge. This was within the range reported in a twoinstitution study in which tracheostomies in preterm infants were placed at an average age of 3-5 months of age [4]. Three subjects with tracheostomies placed after NICU discharge who were included in this analysis.

Our study found a higher proportion of patients of Black race had tracheostomy placed. This is particularly interesting given that infants of Black race are more likely to be born preterm [30] yet have lower rates of both respiratory distress syndrome and BPD $[31,32]$. A recent large prospective study of 458,624 very low birthweight infants born at 796 North American centers found that African American maternal race was an independent risk factor associated with tracheostomy placement [33]. The increased rate of tracheostomy placement in Black infants in this study could be related to increased late developing respiratory morbidity that can be seen among former preterm infants of Black race $[7,32]$. The Prematurity and Respiratory Outcome Program assessed 707 former preterm infants born at $<29$ weeks, finding a lower rate of BPD among Black infants, but increased first year respiratory medication use, symptoms, and hospitalizations $(79 \%$ 
Black and $63 \%$ White). Another factor could be related to social determinants of health that influence the discussion around placement. A recent review of racial and ethnic disparities in NICU care noted that Black families reported that their requests and concerns were commonly dismissed, their decision making capacity was questioned, and that they did not feel part of the care process [34]. The experience of families from different racial and cultural backgrounds surrounding tracheostomy placement is not well described and deserves more investigation in the future.

We found that former tBPD subjects had lower lung function based on $\mathrm{FEV}_{1} \mathrm{pp}$ and $\mathrm{FEV}_{1} / \mathrm{FVC}$ compared to $\mathrm{SBPD}$ subjects. There is limited research on the effects of history of tracheostomy for BPD patients on PFT outcomes [3]. Cristea et al. examined a cohort of 19 tBPD subjects whose PFT measurements showed severe obstructive lung disease that persisted over time, though this was not compared to SBPD [35]. By performing this comparison, we are able to draw conclusions about what might influence the decreased lung function found in the tBPD population, including the impact of subglottic stenosis.

In the visual assessment of the flow-volume loops we noted a subset of tBPD subjects with a reproducible pattern of flattened expiratory curves, which lacked an expiratory peak and had a flattened slope. The FEV 1 in these studies appeared to be reduced from upper airway obstruction, rather than lower respiratory disease. A possible explanation for this is the increased diagnoses of subglottic stenosis in the tBPD subjects, which can either be a reason for or consequence of the tracheostomy $[25,36]$. Overall in this study $63.3 \%$ of tBPD subjects had subglottic stenosis; and among tBPD patients with subglottic stenosis, $21.1 \%$ exhibited flattened expiratory curves. In studies of long-term lung function in BPD that include former tracheostomy subjects, these flattened loops may make it appear that these subjects have lower respiratory function by decreasing the FEV based on the upper respiratory disease rather than lower respiratory disease. Investigators should use caution when interpreting decreases in $\mathrm{FEV}_{1}$ for these subjects.

In this cohort, tBPD patients had lower scores for cognitive, gross motor, and fine motor development at 12-23.99 months CGA, and lower cognitive scores at 2-3 years CGA. This is in concordance with earlier studies, which have shown more neurodevelopmental impairment in those with more severe BPD $[12,37]$. Interestingly, Cammack et al. examined Bayley cognitive and language scores at $2-3$ years of age among a cohort of 56 severe BPD patients with and without tracheostomy, finding no significant difference between the two, though they noted that both groups had profound impairment in all scales of development [13]. It may be that severe respiratory morbidity directly impacts neurodevelopment or perhaps SBPD and neurocognitive delays are independent outcomes related to a common prior antecedent associated with preterm birth. In our overall cohort, we found that post-natal steroids were administered to a greater proportion of tBPD subjects than SBPD subjects, which could contribute to increased levels of NDI seen in tBPD subjects [38]. Further analyses with longitudinal data in larger cohorts is needed to address these questions [39].

Our study represents severe BPD subjects from a single tertiary/ quaternary care center with a large referral base among regional NICUs and a high rate of sBPD. This is a strength but may limit applicability as local resources and practices vary widely. The tBPD subjects had earlier birth years and there may have been in advances in technology and standards of care that are potential confounders. We were able to extract a large amount of retrospective information about the NICU course; however, there were missing data as not all care was received within our institution not all outside records were available. This made it difficult to ascertain the indication for tracheostomy and other details. It also limited our ability to examine post-NICU data including emergency visits and readmissions. Additionally, only a subset of subjects returned for pulmonary function and developmental testing, which limited the proportion of the cohort with these follow-up studies particularly among sBPD subjects; tBPD subjects were more likely to continue to follow in pulmonary clinic at older ages due to their illness severity. Ideally, we would have examined the overlapping respiratory and neurodevelopmental outcomes, however among tBPD subjects only six had both tests.

In comparing long-term outcomes among this large cohort of former preterm infants with severe BPD, we found decreased lung function and more neurodevelopmental impairment among those with $\mathrm{tBPD}$, which is likely related to the severity of lung disease and associated comorbidities rather than being driven by the tracheostomy itself. Future studies should be aimed at examining the complicated relationship between long-term respiratory and cognitive impairments in severe BPD. We found that Black race was associated with increased likelihood of tracheostomy placement in severe BPD, which could be driven social determinants of health and their physiologic manifestations. Among those with tBPD, we found that subglottic stenosis may be a reason for lung function to appear reduced by spirometry measurement, while actually representing a component of upper airway disease, a factor that should be considered when examining lung function in this population. By directly comparing SBPD and tBPD subjects in this cohort, this study demonstrates the impact of greater disease severity among the tBPD population, which can inform decision making and help providers and families understand the potential outcomes for this extreme population with tBPD.

\section{REFERENCES}

1. Abman SH, Collaco JM, Shepherd EG, Keszler M, Cuevas-Guaman M, Welty SE, et al. Interdisciplinary care of children with severe bronchopulmonary dysplasia. J Pediatr. 2017;181:12-28. e11

2. Sakai M, Kou YF, Shah GB, Johnson RF. Tracheostomy demographics and outcomes among pediatric patients ages 18 years or younger-United States 2012. Laryngoscope. 2019;129:1706-11.

3. Koltsida G, Konstantinopoulou S. Long term outcomes in chronic lung disease requiring tracheostomy and T chronic mechanical ventilation. Semin Fetal Neonatal Med. 2019;24:101044.

4. Pereira KD, MacGregor AR, McDuffie CM, Mitchell RB. Tracheostomy in preterm infants: current trends. Arch Otolaryngol Head Neck Surg. 2003;129:1268-71.

5. Higgins RD, Jobe AH, Koso-Thomas M, Bancalari E, Viscardi RM, Hartert TV, et al. Bronchopulmonary dysplasia: executive summary of a workshop. J Pediatr. 2018;197:300-8.

6. Wu KY, Jensen EA, White AM, Wang Y, Biko DM, Nilan K, et al. Characterization of disease phenotype in very preterm infants with severe bronchopulmonary dysplasia. Am J Respir Crit Care Med. 2020;201:1398-406.

7. Keller RL, Feng R, DeMauro SB, Ferkol T, Hardie W, Rogers EE, et al. Bronchopulmonary dysplasia and perinatal characteristics predict 1-year respiratory outcomes in newborns born at extremely low gestational age: a prospective cohort study. J Pediatr. 2017;187:89-97. e83

8. Fawke J, Lum S, Kirkby J, Hennessy E, Marlow N, Rowell V, et al. Lung function and respiratory symptoms at 11 years in children born extremely preterm: the EPICure study. Am J Respir Crit Care Med. 2010;182:237-45.

9. Vom Hove $M$, Prenzel $F$, Uhlig $H$, Robel-Tillig E. Pulmonary outcome in former preterm, very low birth weight children with bronchopulmonary dysplasia: a case-control follow-up at school age. J Pediatr. 2014;164:40-45. e44

10. Verheggen M, Wilson A, Pillow J, Stick S, Hall G. Respiratory function and symptoms in young preterm children in the contemporary era. Pediatr Pulmonol. 2016;51:1347-55.

11. Kaslovsky R, Sadof M. Spirometry for the primary care pediatrician. Pediatrics Rev. 2014;35:465-75.

12. Brumbaugh JE, Bell EF, Grey SF, DeMauro SB, Vohr BR, Harmon HM, et al. Behavior profiles at 2 years for children born extremely preterm with bronchopulmonary dysplasia. J Pediatr. 2020;219:152-9.

13. Cammack B, Noel-MacDonnell J, Cuna A, Manimtim W. Impact of tracheostomy on language and cognitive development in infants with severe bronchopulmonary dysplasia. J Perinatol. 2020;40:299-305.

14. Cristea Al, Carroll AE, Davis SD, Swigonski NL, Ackerman VL. Outcomes of children with severe bronchopulmonary dysplasia who were ventilator dependent at home. Pediatrics. 2013;132:e727-734.

15. Levin JC, Sheils CA, Gaffin JM, Hersh CP, Rhein LM, Hayden LP. Lung function trajectories in former premature infants: identifying risk factors for abnormal lung growth. Respir Res. 2021;22:143. 
16. Goryachev S, Sordo M, Zeng QT. A suite of natural language processing tools developed for the I2B2 project. AMIA Annu Symp Proc. 2006;2006:931.

17. Jobe AH, Bancalari E. Bronchopulmonary dysplasia. Am J Respir Crit Care Med. 2001;163:1723-9.

18. HoundDog. Research computing. Boston: Boston Children's Hospital; 2018.

19. Miller MR, Hankinson J, Brusasco V, Burgos F, Casaburi R, Coates A, et al. Standardisation of spirometry. Eur Respir J. 2005;26:319-38.

20. Quanjer PH, Stanojevic S, Cole TJ, Baur X, Hall GL, Culver BH, et al. Multi-ethnic reference values for spirometry for the 3-95-yr age range: the global lung function 2012 equations. Eur Respir J. 2012;40:1324-43.

21. Bayley N. Bayley scales of infant and toddler development. 3rd ed. San Antonio, TX: Harcourt Assessment; 2006.

22. Corp. I IBM SPSS Statistics for Macintosh. 26.0 ed. Armonk, NY: IBM Corp.; 2018.

23. Carron JD, Derkay CS, Strope GL, Nosonchuk JE, Darrow DH. Pediatric tracheotomies: changing indications and outcomes. Laryngoscope. 2000;110:1099-104.

24. Levit OL, Shabanova V, Bazzy-Asaad A, Bizzarro MJ, Bhandari V. Risk factors for tracheostomy requirement in extremely low birth weight infants. J Matern-Fetal Neonatal Med. 2018;31:447-52.

25. Grundfast KM, Felizardo S, Camilon J, Barber CS, Pransky S, Fink R. Prospective study of subglottic stenosis in intubated neonates. Ann Otol Rhinol Laryngol. 1990;99:390-5.

26. Lee JH, Smith PB, Quek MBH, Laughon MM, Clark RH, Hornik CP. Risk factors and inhospital outcomes following tracheostomy in infants. J Pediatr. 2016;173:39-44.

27. Lewis CW, Carron JD, Perkins JA, Sie KCY, Feudtner C. Tracheotomy in pediatric patients: a national perspective. Arch Otolaryngol Head Neck Surg. 2003;129:523-9.

28. Stark AR, Eichenwald EC. Bronchopulmonary dysplasia: management. UpToDate 2019 February 7, 2019 [cited 2020 June 18]. Available from: https://www. uptodate.com

29. Guaman MC, Gien J, Baker CD, Zhang H, Austin ED, Collaco JM. Point prevalence, clinical characteristics, and treatment variation for infants with severe bronchopulmonary dysplasia. Am J Perinatol. 2015;32:960-7.

30. Thoma ME, Drew LB, Hirai AH, Kim TY, Fenelon A, Shenassa ED. Black-White disparities in preterm birth: geographic, social, and health determinants. Am J Prev Med. 2019;57:675-86.

31. Kavvadia V, Greenough A, Dimitriou G, Hooper R. Influence of ethnic origin on respiratory distress syndrome in very premature infants. Arch Dis Child Fetal Neonatal Ed. 1998;78:F25-28.

32. Ryan RM, Feng R, Bazacliu C, Ferkol TW, Ren CL, Mariani TJ, et al. Black race is associated with a lower risk of bronchopulmonary dysplasia. J Pediatr. 2019;207:130-5. e132

33. Han SM, Watters KF, Hong CR, Edwards EM, Knell J, Morrow KA, et al. Tracheostomy in very low birth weight infants: a prospective multicenter study. Pediatrics. 2020;145:e20192371.

34. Sigurdson K, Mitchell B, Liu J, Morton C, Gould JB, Lee HC, et al. Racial/ethnic disparities in neonatal intensive care: a systematic review. Pediatrics. 2019;144: e20183114.

35. Cristea Al, Ackerman VL, Swigonski NL, Yu Z, Slaven JE, Davis SD. Physiologic findings in children previously ventilator dependent at home due to bronchopulmonary dysplasia. Pediatr Pulmonol. 2015;50:1113-8.
36. Contecin P, Narcy P. Size of endotracheal tube and neonatal acquired subglottic stenosis. Arch Otolaryngol Head Neck Surg. 1993;119:815-9.

37. Ehrenkranz RA, Walsh MC, Vohr BR, Jobe AH, Wright LL, Fanaroff AA, et al. Validation of the National Institutes of Health consensus definition of bronchopulmonary dysplasia. Pediatrics. 2005;116:1353-60.

38. Doyle LW, Davis PG, Morley CJ, McPhee A, Carlin JB. Outcome at 2 years of age of infants from the DART study: a multicenter, international, randomized, controlled trial of low-dose dexamethasone. Pediatrics. 2007;119:716-21.

39. Litt JS, Minich N, Taylor HG, Tiemeier H. The inter-relationships of extremely low birth weight, asthma, and behavior: a study of common cause, mediation, and moderation. Acad Pediatr. 2020;20:975-82.

\section{ACKNOWLEDGEMENTS}

Lawrence M. Rhein, MD MPH, Department of Pediatrics, University of Massachusetts, Worcester, MA, United States.

\section{AUTHOR CONTRIBUTIONS}

C.A.A. worked on the design of the study, conducted the analysis and drafted the initial manuscript. J.C.L. aided with the conceptualization of the study, assisted with statistical analysis, and reviewed the manuscript. J.S.L. helped design the methodology and reviewed the manuscript. C.A.S. aided with investigation, provided resources, and reviewed the manuscript. L.P.H. provided supervision for the design, methodology, and analysis and provided multiple manuscript revisions.

\section{FUNDING}

Supported by the National Institutes of Health (NHLBI K23 HL136851 [to L.P.H.], NICHD K23 HD088695 [to J.S.L.], NLM T15LM007092 [to N. Gehlenborg])

\section{COMPETING INTERESTS}

The authors declare no competing interests.

\section{ADDITIONAL INFORMATION}

Supplementary information The online version contains supplementary material available at https://doi.org/10.1038/s41372-021-01144-0.

Correspondence and requests for materials should be addressed to L.P.H.

Reprints and permission information is available at http://www.nature.com/ reprints

Publisher's note Springer Nature remains neutral with regard to jurisdictional claims in published maps and institutional affiliations. 